\title{
Sistemas granulares aerobios para el tratamiento descentralizado de aguas servidas y su reutilización en condominios en Chile
}

\author{
Granular aerobic systems: viable technology for wastewater \\ treatment and its reuse for Chilean condominium
}

\author{
Natalia Araneda ${ }^{1} \quad$ Daniel Valenzuela-Heredia ${ }^{1} \quad$ José Luis Campos $^{1 *}$ \\ Paula Carrera $^{2} \quad$ Marisol Belmonte $^{3} \quad$ Anuska Mosquera-Corral $^{2} \quad$ Ángeles Val de Río ${ }^{2}$
}

Recibido 17 de Mayo de 2018, Received: May 17, 2018

Aceptado 31 de enero de 2019 Accepted: January 31, 2019

\begin{abstract}
RESUMEN
En este trabajo se propone una alternativa de tratamiento in-situ descentralizado de aguas residuales procedentes de condominios basada en un sistema compacto de biomasa granular aerobia, con el fin de obtener efluentes reutilizables. La operación de un reactor a escala laboratorio mostró que era posible obtener biomasa aerobia granular tratando este tipo de efluente. El proceso de granulación se obtuvo a los 14 días de operación, alcanzando porcentajes de eliminación de materia orgánica del 75-90\%. Basándose en la información obtenida experimentalmente con un reactor de laboratorio se ha realizado el diseño de una planta capaz de tratar agua residual de un condominio de 200 personas, siendo necesaria un área de implantación de $25,5 \mathrm{~m}^{2}$. Por último se evaluaron dos modelos de negocio consistentes en dos alternativas de reutilización del agua tratada: (i) venta de agua a la Municipalidad o (ii) su infiltración en las napas subterráneas. Ambas opciones son económicamente viables, siendo la primera la que más ingresos genera.
\end{abstract}

Palabras clave: Aguas servidas, reutilización, sistemas granulares aerobios.

\begin{abstract}
An alternative of decentralized in-situ treatment for wastewater from condominiums based on aerobic granular biomass is proposed in this research to promote water reuse. It was demonstrated at a laboratory scale that it is possible to achieve granular biomass with this kind of wastewater. The granulation process was completed after 14 days of operation, achieving COD removal percentages of 75-90\%. A compact design of a pilot plant was made to treat wastewater from a condominium of 200 people. The needed implantation area was $25.5 \mathrm{~m}^{2}$. Finally, two different alternatives of business models were studied: selling the treated water to the municipal organism or infiltrate it into groundwater. Between both options, selling the treated water allows a higher income and makes it a better alternative.
\end{abstract}

Keywords: Wastewater, reuse, granular aerobic systems.

\footnotetext{
1 Universidad Adolfo Ibáñez de la Universidad. Facultad de Ingeniería y Ciencias. Viña del Mar, Chile. E-mail: naraneda@outlook.com; daniel.valenzuela@edu.uai.cl, jluis.campos@uai.cl

2 Universidad de Santiago de Compostela. Departamento de Ingeniería Química. Santiago de Compostela, España.

E-mail: paula.carrera@usc.es, mangeles.val@usc.es, anuska.mosquera@usc.es

3 Universidad de Playa Ancha. Facultad de Ingeniería. Valparaíso, Chile. E-mail: marisol.belmonte@upla.cl

* Autor de correspondencia: jluis.campos@uai.cl
} 


\section{INTRODUCCIÓN}

El aumento de la población y de los niveles de contaminación junto con el cambio climático hacen que sea necesario una gestión del agua cada vez más sustentable [1]. En este sentido, en muchos países se está considerando a las aguas residuales como un recurso hídrico a tener en cuenta. De hecho, la obtención de agua potable a partir de aguas residuales se está llevando a cabo en distintos lugares como Singapur, Estados Unidos, Israel y Namibia entre otros y se ha comprobado que esta opción es económicamente más viable que el uso de plantas desaladoras [2]. Una gestión adecuada de las aguas residuales con el fin de poderla reutilizar no es posible algunas veces debido a vacíos administrativos. Sin embargo, las normativas respecto a dicha reutilización deberían de ir cambiando con el fin de adaptarse a nuevos escenarios de escasez hídrica y a la aparición de nuevas tecnologías para el tratamiento como es el caso de Chile.

En Chile, las aguas residuales son enviadas a través de sistemas de alcantarillado a plantas de tratamiento centralizadas, que optan comúnmente por sistemas de lodos activos para la eliminación de materia orgánica. El uso de estos sistemas centralizados presenta una serie de desventajas, entre las que destaca la imposibilidad de reutilización del agua tratada en las ciudades de procedencia debido a los altos costes económicos que implicaría su posterior bombeo. Por este motivo, la descentralización del tratamiento de aguas parece una alternativa a plantear con el fin de reducir los costes del agua de cara a su reutilización y de favorecer el posible aprovechamiento de los nutrientes presentes como fertilizante, evitando así el uso de agua potable [3].

Un posible nicho de aplicación de estos sistemas de tratamiento descentralizados son los condominios que representan un papel importante en la forma en que se distribuye la población chilena pues concentran más de un millón de habitantes de los 17,6 millones que constituyen la población del país. Estos condominios son núcleos poblacionales dispersos que, a pesar de su relevancia en términos de población, no suelen disponer de un sistema propio de tratamiento de aguas residuales. Debido a los bajos costes de implantación y a su relativa sencillez de operación una alternativa para el tratamiento descentralizado de estas aguas residuales es la utilización de humedales artificiales [4], tal y como muestran experiencias realizadas en Israel [5] y Chile [6]. Estos sistemas permiten obtener un efluente con una calidad adecuada para su reutilización en agricultura [7-8]. No obstante, la elevada superficie de implantación requerida por los humedales artificiales hace que no sea factible su aplicación en condominios por lo que esta quedaría más bien relegada a zonas rurales. Por otro lado, aunque la tecnología de tratamiento de aguas residuales más comúnmente usada en los sistemas centralizados es la de lodos activos, su aplicación en condominios sería inviable debido también al gran requerimiento de área para su implantación como consecuencia de las malas características de sedimentabilidad de la biomasa [9]. Dentro de las tecnologías alternativas, los sistemas basados en biomasa granular aerobia son a priori una opción adecuada para realizar in-situ el tratamiento de las aguas residuales de los condominios dado que su área de implantación es un $25 \%$ de la requerida por los sistemas de lodos activos [10-11] y el consumo energético se podría reducir del $65-75 \%$ [12]. En relación con otros sistemas de tratamiento compacto como pueden ser los reactores de biopelículas o los biorreactores de membranas, los sistemas basados en biomasa granular aerobia presentan un ahorro energético del 35 al 70\% [13]. En trabajos de investigación realizados por $\mathrm{Ni}$ et al. [14] y Pronk et al. [15] se demostró que es posible tratar agua residual urbana utilizando biomasa granular aerobia. La implantación de este tipo de sistemas permitiría eliminar gastos operacionales de bombeo del agua residual hasta las plantas centralizadas, evitar su posible mezclado con aguas industriales y/o pluviales y posibles emisiones gaseosas durante su transporte, reducir los gastos asociados al tratamiento de aguas residuales a los propios condominios y hacer posible la reutilización del agua tratada, bien para regadío o para su venta a otras entidades [16].

Con el objetivo de comprobar la viabilidad económica de la tecnología granular aerobia para el tratamiento descentralizado de aguas residuales procedentes de condominios, se estudiaron dos modelos de negocio que dan un uso diferente al agua tratada: venta a la municipalidad e infiltración subterránea. El diseño de la planta se realizó en base a resultados obtenidos a escala laboratorio. 


\section{MATERIALES Y MÉTODOS}

\section{Reactor a escala laboratorio}

Con el fin de obtener información básica para el posterior escalado se operó un reactor secuencial SBR (Sequencing Batch Reactor) con un volumen útil de $3 \mathrm{~L}$ (altura útil de $50 \mathrm{~cm}$ y diámetro de $10 \mathrm{~cm}$ ) (Figura 1). La relación de intercambio volumétrico fue del $50 \%$ y la duración de los ciclos de operación fue de 3 horas. Los ciclos constaron de las siguientes etapas (Figura 2): alimentación, reacción en condiciones aerobias, sedimentación, vaciado del reactor y parada. La duración de las fases de aireación y sedimentación se modificó a lo largo del periodo de operación del reactor, de forma que durante la puesta en marcha el período de sedimentación fue mayor para evitar una excesiva pérdida de biomasa, y se redujo una vez que se completó la granulación de la biomasa para mejorar su selección.

El caudal de aire suministrado durante la fase de reacción fue de $9,2 \mathrm{~L} / \min \left(55,2 \mathrm{~kg} \mathrm{O}_{2} / \mathrm{m}^{3}\right.$ reactor $\left.\times \mathrm{h}\right)$, alcanzándose concentraciones de oxígeno disuelto superiores a $5 \mathrm{mg} / \mathrm{L}$ durante prácticamente todo el ciclo. El reactor se alimentó con un medio sintético con características similares al agua residual producida en los condominios (500 $\mathrm{mg} / \mathrm{L}$ de DQO (Demanda Química de Oxígeno); 50 mg N/L) [17].
El inóculo empleado para poner en marcha el reactor fue lodo activo floculento (relación entre sólidos en suspensión volátiles y totales (SSV/SST): 0,8; índice volumétrico de lodos (IVL): $152 \mathrm{~mL} / \mathrm{g} \mathrm{SST}$ ) procedente de una planta de tratamiento de aguas residuales de la empresa Aguas Andinas situada en la localidad de Curacaví (Región de Valparaíso, Chile).

El reactor se operó durante 80 días con las condiciones de operación descritas anteriormente, lo que permitió imponer unas condiciones hidráulicas de estrés (cortos tiempos de sedimentación y elevado intercambio volumétrico en cada ciclo) suficientes para llevar a cabo el proceso de granulación. En este tiempo de operación se realizó un seguimiento de la fase líquida (el licor de mezcla y el efluente) y la fase sólida. En

\begin{tabular}{|l|c|c|c|c|}
\hline \multicolumn{1}{|c|}{ Etapa } & \multicolumn{5}{c|}{ Tiempo (min) } \\
\hline Alimentación & 5 & & 1 & \\
\hline Aireación & \multicolumn{2}{|c|}{170} & 3 & \\
\hline Decantado & \multicolumn{5}{|c|}{180} \\
\hline Vaciado & & & 1 \\
\hline Parada & \multicolumn{5}{|c|}{180} \\
\hline Total ciclo & \multicolumn{5}{|c|}{} \\
\hline
\end{tabular}

Figura 2. Distribución de un ciclo de operación del reactor granular durante la etapa de operación.

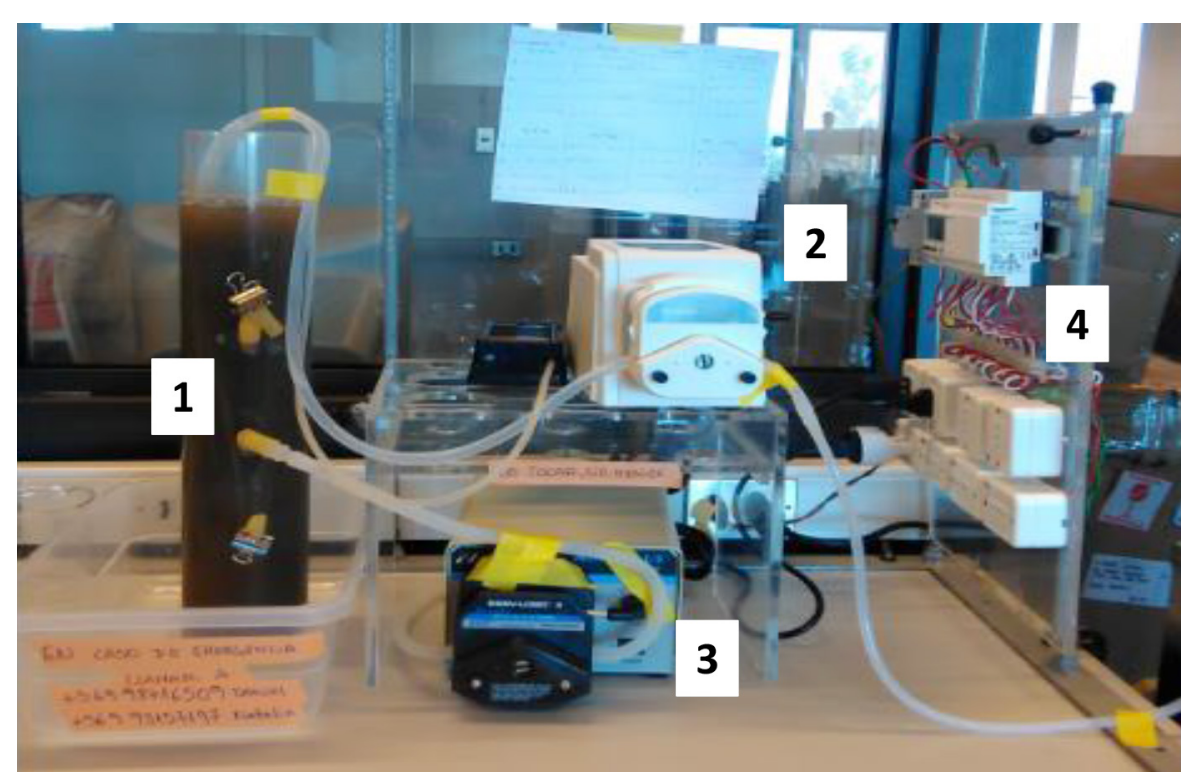

Figura 1. Montaje experimental: 1) Reactor aerobio de biomasa granular; 2) bomba de alimentación; 3) bomba de vaciado; 4) sistema de control. 
la fase líquida se analizaron las concentraciones de la demanda química de oxígeno (DQO), amonio, nitrito y nitrato mediante kits de HACH. Se realizó también una monitorización de la concentración de oxígeno disuelto, temperatura y $\mathrm{pH}$ a lo largo de los ciclos. Para la caracterización de la fase sólida se determinó el contenido de sólidos en suspensión totales (SST) en el reactor y el efluente, el índice volumétrico de lodos a los 30 minutos $\left(\mathrm{IVL}_{30}\right)$ y el tamaño de los gránulos de acuerdo a los protocolos establecidos en el Standards Methods [18].

\section{Diseño de la planta y cálculo de los costes de operación y capital}

Para llevar a cabo el dimensionamiento de la planta de tratamiento se tomó como base un condominio de 50 viviendas, con un total de 200 habitantes. Dado que el caudal promedio de aguas residuales producido en Chile por habitante es de $140 \mathrm{~L} / \mathrm{d}$ [19], se diseñó la planta para tratar un caudal de entrada de $28 \mathrm{~m}^{3} / \mathrm{d}$.

La integración de las distintas unidades que componen la planta se realizó con el programa Rhinoceros con el fin de obtener un sistema lo más compacto posible para evitar posibles restricciones de terreno y también para poder recolectar las corrientes gaseosas generadas y tratarlas, evitando así problemas de olores.

Costes de operación: Para determinar los costes de operación asociados a la aireación y gestión de lodos, se realizó el cálculo del oxígeno consumido y de los lodos generados en base a balances de materia y se tomó un valor de 0,09 US\$/kWh y costo de gestión de 37,46 US\$/tonelada de lodo [20]. La determinación de los costos de bombeo se realizó considerando la potencia de la bomba $(\mathrm{kW})$, las horas de operación y el costo de la energía mencionado anteriormente. El costo de insumos tales como carbón activo y cloro se hizo teniendo en cuenta su precio unitario de mercado y la vida útil del carbón activo y la adición de una dosis de $2,0 \mathrm{mg} / \mathrm{L}$ de cloro. Se consideró que se emplearía a una persona que supervisaría la planta dos días a la semana (5 h/d) con un sueldo 3,60 US\$/h. Los costos anuales de seguimiento de la operación relativos a análisis químicos y biológicos para controlar la calidad del efluente fueron de US\$288,2. Los costes relativos a mantenimiento y seguros se estimaron en base al total de los costes de operación de los equipos, considerando porcentajes del 10 y 5\%, respectivamente.

Costes de capital: Una vez dimensionadas las distintas unidades de la planta los costes de capital se calcularon en base a referencias bibliográficas (rejas de desbaste [21]; reactores SBR [22]; soplante [23]; tanque tampón [24]; tanque de cloración/sedimentador [25]; bombas [26]) y datos de mercado (filtro de carbón activo, prensa manual de lodos, tanque de almacenamiento de lodos y tubos Venturi). Además de tener en cuenta los costos de los equipos, se determinaron otros costos de inversión necesarios para llevar a cabo el proyecto a partir de factores de inversión, que son porcentajes de la inversión total realizada para los equipos. Para las tuberías, válvulas y accesorios se consideró un factor de inversión de $20 \%$, para control e instrumentos de $15 \%$, para la instalación eléctrica de $20 \%$, para la seguridad y salud de $5 \%$ y para la obra civil de $20 \%$ [23].

\section{Evaluación de los modelos de negocio}

Se plantearon dos modelos de negocio: el primero consistía en el uso de agua para el regadío de los condominios y la venta a la Municipalidad del agua restante. El segundo modelo tendría como objetivo el uso del agua tratada para el regadío y la infiltración del agua excedente en las napas subterráneas para recuperar los acuíferos, lo cual estaría subvencionado parcialmente por el Gobierno.

La evaluación de los modelos de negocio se llevó a cabo mediante el cálculo del VAN (Valor Actual Neto), el TIR (Tasa Interna de Retorno) y el tiempo necesario para recuperar la inversión. El análisis de sensibilidad, para evaluar la influencia del precio del agua, el precio de la venta del agua, la energía y los impuestos sobre el valor del VAN, fue realizado con la herramienta Oracle, Crystal Ball como una extensión del programa Microsoft Excel.

\section{RESULTADOS Y DISCUSIÓN}

\section{Operación del reactor aerobio granular a escala laboratorio}

Durante todo el periodo de operación la eliminación de materia orgánica alcanzó valores del 75-90\% a una velocidad de carga orgánica aplicada de $2 \mathrm{~kg} \mathrm{DQO} / \mathrm{m}^{3} \cdot \mathrm{d}$ (Figura 3(A)) mientras que la eliminación de amonio se mantuvo en un $40 \%$. 

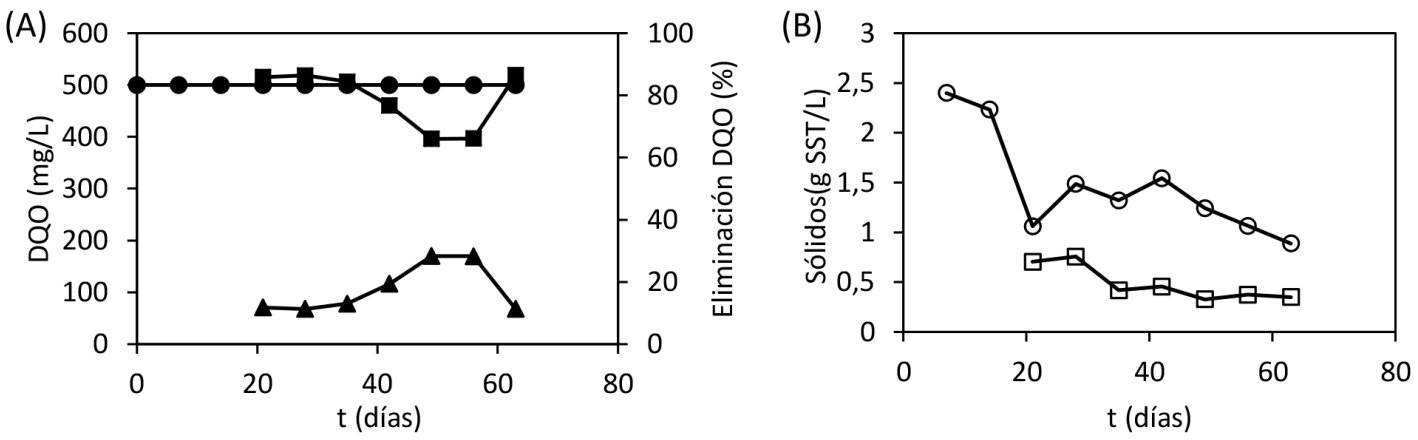

Figura 3. (A) Evolución de la concentración de DQO (mg/L) en el influente ( $)$, efluente (A) y porcentaje de eliminación de DQO (ם) durante la operación del reactor. (B) Evolución de la concentración de sólidos en el efluente $(\square)$ y en el interior del reactor $(\circ)$.

Dado que no se detectó la presencia de nitrito ni nitrato durante el seguimiento de los ciclos de operación, la eliminación de amonio es atribuible a su asimilación para el crecimiento de la biomasa. La ausencia de bacterias nitrificantes está asociada a los bajos valores de tiempo de retención celular (TRC) a los que se operó el sistema (1-2 d). Este retardo en el desarrollo de biomasa nitrificante es habitualmente observado en sistemas granulares aerobios debido a un excesivo lavado de biomasa durante las primeras semanas de operación [25] y al lento crecimiento de las bacterias nitrificantes. Por tanto, para asegurar el crecimiento de bacterias nitrificantes en la biomasa granular aerobia, sería necesario un tiempo de operación más prolongado que permitiera aumentar el TRC.

Debido al estrés hidráulico impuesto dentro del reactor los primeros agregados de biomasa aparecieron el día 7 de operación. El día 14 se completó el proceso de granulación, obteniendo gránulos perfectamente esféricos el día 17. Durante los primeros días de operación la concentración de biomasa disminuyó de 2,5 g SST/L a un valor de 1-1,5 g SST/L (Figura 3(B)). Este valor se mantuvo constante el resto de la operación dado que se realizó una purga selectiva de los gránulos de mayor tamaño, ya que su menor área específica limita la transferencia de oxígeno, causando un detrimento en la eficacia de eliminación. La biomasa granular obtenida tuvo un diámetro medio de $0,88 \mathrm{~cm}$ (Figura 4) y un $\mathrm{IVL}_{30}$ entre $95-120 \mathrm{~mL} / \mathrm{g}$ SST. El valor del $\mathrm{IVL}_{30}$ superior a $60-80 \mathrm{~mL} / \mathrm{g}$ SST indica unas pobres propiedades de sedimentación de los agregados para ser biomasa granular aerobia, por lo que el proceso de granulación aerobia no estaba consolidado.

En base a los resultados operacionales presentados, se puede afirmar que es posible obtener biomasa granular aerobia a partir de lodo activo en un corto período de tiempo tratando aguas residuales procedentes de condominios. Sin embargo, es necesario optimizar la operación del sistema en

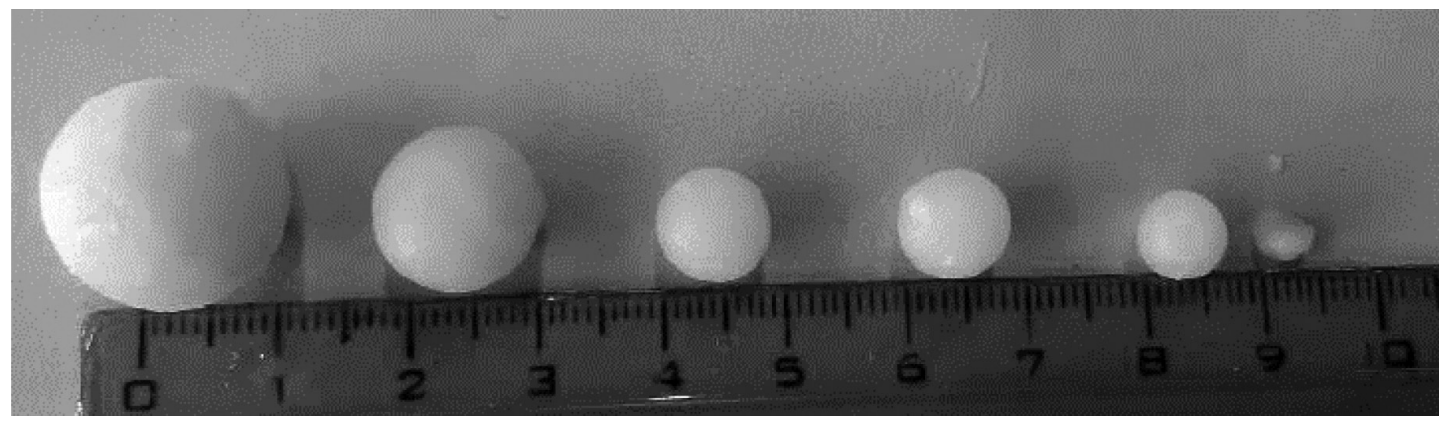

Figura 4. Gránulos formados durante la operación del reactor. 
caso de que se necesitara eliminar compuestos nitrogenados, por ejemplo aumentando el TRC para favorecer el desarrollo de bacterias nitrificantes y configurando el ciclo operacional para disponer de una fase anóxica que permita potenciar la desnitrificación.

\section{Diseño de la planta de tratamiento}

En base al caudal a tratar y a las características del agua residual, se realizó el diseño de una planta basada en la tecnología granular aerobia, que cumpliese con las normativas de vertido, con el objetivo de estudiar su viabilidad en base a los costes asociados y las necesidades de espacio. Cabe resaltar que en este caso no se ha tenido en cuenta en el diseño la eliminación de contaminantes emergentes, aunque su creciente importancia se debería tener en cuenta en el futuro debido a un posible cambio de legislación que implique su eliminación. En la Tabla 1 se describen los parámetros de diseño de cada una de las unidades.

Línea de aguas: La línea de aguas cuenta con tres tipos de procesos encargados de tratar el agua residual producida en el condominio: pretratamientos, tratamiento biológico y postratamientos. En principio es necesario un pretratamiento del agua residual para la eliminación de sólidos y regular el caudal antes de su entrada al reactor granular. Este pretratamiento está constituido por dos unidades. La primera consiste en rejas de desbaste finas, encargadas de eliminar los sólidos de tamaño superior a $6 \mathrm{~mm}$ presentes en el influente. A continuación, un tanque tampón de $4 \mathrm{~m}^{3}$ homogeneiza el caudal de agua residual que entrará a los reactores granulares, ya que el sistema no opera de forma continua.

Una vez reducido el contenido de sólidos, el siguiente paso es la eliminación de la materia orgánica y los nutrientes mediante procesos biológicos en el reactor granular. Este tiene lugar en dos reactores SBR con biomasa granular de $3,5 \mathrm{~m}^{3}$, cada uno, que operan en paralelo en ciclos de 3 horas y con una relación de intercambio volumétrico del $50 \%$. Los reactores fueron diseñados de forma que cada uno trata la mitad del caudal de entrada $\left(14 \mathrm{~m}^{3} / \mathrm{d}\right)$ a una velocidad de carga orgánica de $2 \mathrm{~kg}$ DQO $\mathrm{m}^{3} \cdot \mathrm{d}$. El suministro de aire se realiza a través de una soplante con un caudal de $200 \mathrm{~m}^{3} / \mathrm{d}$.

La última unidad de la línea de aguas consiste en un tanque de recepción, sedimentación y cloración, encargado de acondicionar el agua para cumplir las especificaciones requeridas para su posterior uso, incluyendo su desinfección y reducción del contenido en sólidos. El volumen de esta unidad es de $2 \mathrm{~m}^{3}$.

Línea de lodos: La línea de lodos cuenta con una prensa manual para deshidratar el lodo procedente de los reactores biológicos, del tanque de cloración y de las rejas de desbaste con el fin de obtener una sequedad del $25 \%$. Los lodos deshidratados se

Tabla 1. Resumen de las unidades necesarias en el proceso.

\begin{tabular}{|c|c|c|c|c|}
\hline Proceso & Objetivo & Unidad & Volumen & Parámetro operacional \\
\hline \multirow[b]{2}{*}{ Pretratamiento } & Elim. de sólidos & Rejas de desbaste & - & Área efectiva de apertura de $0,6 \mathrm{~m}^{2}$ \\
\hline & $\begin{array}{l}\text { Homogeneización } \\
\text { del influente de los } \\
\text { reactores }\end{array}$ & Tanque tampón & $4 \mathrm{~m}^{3}$ & Caudal promedio de $1,167 \mathrm{~m}^{3} / \mathrm{h}$ \\
\hline Reactor biológico & Elim. M.O. y N & 2 reactores granulares & $2 \times 3,5 \mathrm{~m}^{3}$ & $\begin{array}{l}\text { Elim. DQO (75-90\%). } \\
\text { Reducción NT a } 10 \text { mg/L } \\
\text { (valor límite de emisión) }\end{array}$ \\
\hline Post-Tratamiento & $\begin{array}{l}\text { Elim. sólidos y } \\
\text { desinfección }\end{array}$ & Tanque de cloración & $2 \mathrm{~m}^{3}$ & Elim. $99,9 \%$ de virus y bacterias \\
\hline \multirow{2}{*}{ Tratamiento de lodo } & $\begin{array}{l}\text { Reducción de agua } \\
\text { del lodo }\end{array}$ & Filtro prensa & - & Reducción humedad (75\%) \\
\hline & $\begin{array}{l}\text { Almacenamiento } \\
\text { del lodo }\end{array}$ & Tanque de recepción & $0,15 \mathrm{~m}^{3}$ & Producción de 112 kg lodo/semana \\
\hline Tratamiento de gases & Elim. COV & $\begin{array}{l}\text { Filtro de carbón } \\
\text { activo }\end{array}$ & $0,012 \mathrm{~m}^{3}$ & Tiempo de residencia de 5 segundos \\
\hline
\end{tabular}

Elim.: eliminación; M.O.: materia orgánica; N: nitrógeno; NT: Nitrógeno total; COV: compuestos orgánicos volátiles. 
almacenarían en un tanque de recepción de 150 L de donde se retirarían semanalmente.

Línea de gases: La línea de gases transporta el aire de salida de los reactores granulares hacia un filtro de carbón activo de 12 L para la eliminación de posibles compuestos orgánicos volátiles. El tanque de homogeneización y el de lodos estarían cubiertos y sus salidas de gases estarían conectadas a la línea principal mediante tubos Venturi con el fin aprovechar el efecto de aspiración.

Teniendo en cuenta todas las líneas de proceso mencionadas anteriormente, el diagrama de flujo del proceso es el correspondiente al mostrado en la Figura 5.

\section{Implantación}

Una vez establecidas las diferentes líneas de proceso y dimensionadas las unidades se realizó un esquema de la planta de modo que esta sea lo más compacta posible y se pueda conocer el área de terreno necesaria para su implantación (Figura 6). Exceptuando los reactores granulares, las demás unidades de proceso se ubicaron en el interior de una caseta, de $3 \mathrm{~m}$ de ancho y 5,5 $\mathrm{m}$ de largo, mientras que estos, debido a su altura, se situaron fuera, ocupando unos $9 \mathrm{~m}^{2}$. Como resultado, el área de implantación se estimó en $25,5 \mathrm{~m}^{2}$.

\section{Costes de implantación y operación de la planta de tratamiento}

Se realizó una estimación de los costes asociados a cada unidad para poder calcular la inversión total necesaria en la planta, que aparece reflejada en el Tabla 2. No solo se consideraron los costes de los equipos, sino que también se incluyeron aquellos costes asociados a equipos auxiliares.

Se realizó también una estimación de los costes de operación anuales asociados a la planta con el

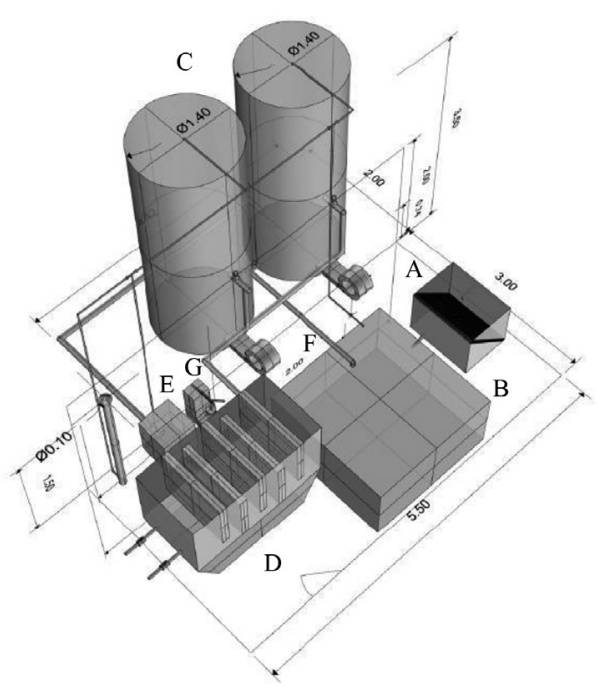

Figura 6. Perspectiva en 3D de la planta piloto de biomasa granular: A) Rejilla de desbaste; B) Tanque tampón; C) Reactores granulares aerobios; D) Tanque de sedimentación/cloración; E) Tanque de almacenamiento de lodos; F) Soplantes; G) Prensa de lodos.

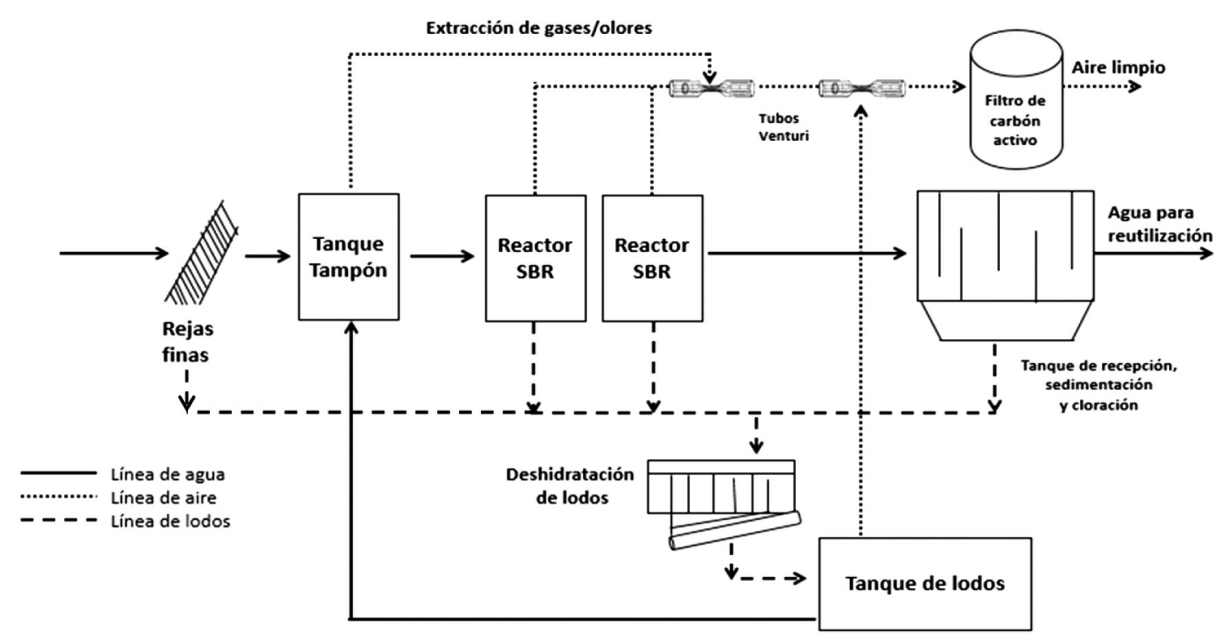

Figura 5. Diagrama de flujo de la planta depuradora con biomasa granular aerobia. 
Tabla 2. Estimación de los costes de inversión de la planta.

\begin{tabular}{|l|r|}
\hline \multicolumn{1}{|c|}{ Unidad } & Coste (US\$) \\
\hline Rejas de desbaste & 835,99 \\
\hline Reactores SBR & $9.596,36$ \\
\hline Soplante & $19.103,68$ \\
\hline Tanque tampón & $6.288,61$ \\
\hline Filtro carbón activado & 288,18 \\
\hline Tanque de cloración/sedimentación & $7.279,80$ \\
\hline Tanque de lodos & 57,64 \\
\hline Bombas hidráulicas & $19.093,06$ \\
\hline Bomba extracción lodos & $5.619,60$ \\
\hline Prensa manual para lodos & $9.077,81$ \\
\hline Tubos Venturi & 32,79 \\
\hline Total inversión en equipos & $77.273,52$ \\
\hline Tuberías, válvulas y accesorios (20\%) & $15.454,70$ \\
\hline Control e instrumentación (15\%) & $11.591,03$ \\
\hline Instalación eléctrica (20\%) & $15.454,70$ \\
\hline Seguridad y Salud (5\%) & $3.863,67$ \\
\hline Obra Civil (20\%) & $15.454,70$ \\
\hline Total inversión & $139.092,32$ \\
\hline
\end{tabular}

objetivo de calcular la rentabilidad de la planta para distintos modelos de negocio (Tabla 3).

\section{Evaluación de los modelos de negocio}

Con el fin de calcular la cantidad de agua tratada de la que se dispondrá para ser vendida, se tuvo en cuenta que, en los condominios, el promedio de áreas verdes por persona es de $4 \mathrm{~m}^{2}$ [26] y que son necesarios $4 \mathrm{~L} / \mathrm{d}$ para regar $1 \mathrm{~m}^{2}$ de área verde [27]. Consecuentemente para un condominio de 200 personas serían necesarios $3,2 \mathrm{~m}^{3} / \mathrm{d}$ del agua tratada en la planta piloto para ser destinados a regadío, mientras que el resto podría ser utilizado para otro fin. En este caso no se ha tenido en cuenta la presencia de compuestos emergentes en las aguas tratadas y se ha considerado que se cumple con la normativa en cuanto a patógenos.

\section{Modelo de negocio 1: Venta del excedente de agua a las municipalidades.}

Actualmente las municipalidades pagan un alto precio por el agua empleada para el regadío, ya que se usa agua potable. De acuerdo a este primer modelo de negocio, el agua tratada se podría vender a la municipalidad a un menor precio que el del agua potable, ya que es un agua apta para regadío al contener nutrientes.
Tabla 3. Costes de operación anuales de la planta.

\begin{tabular}{|l|c|}
\hline \multicolumn{1}{|c|}{ Unidad } & Coste (US\$) \\
\hline Aireación & 250,68 \\
\hline Bombeo & 247,24 \\
\hline Gestión de lodos & 215,67 \\
\hline Total Costes de Operación Equipos & 713,59 \\
\hline Carbón activo & 106,30 \\
\hline Cloración & 220,89 \\
\hline Horas Hombre & $1.921,23$ \\
\hline Seguimiento & 288,18 \\
\hline Mantenimiento (10\%) & 71,36 \\
\hline Seguro (5\%) & 35,68 \\
\hline Total Costes Operacionales & $3.357,23$ \\
\hline
\end{tabular}

Con esta opción el condominio tendría un triple beneficio económico: el ahorro del coste del agua usada para el regadío, el ahorro de los costes de alcantarillado, recolección y tratamiento del agua residual generada y los ingresos por la venta del agua a la Municipalidad. Se realizó un cálculo del ahorro teniendo en cuenta que los costes de alcantarillado, recolección y tratamiento son 0,03 , 0,40 y $0,26 \mathrm{US} \$ / \mathrm{m}^{3}$ respectivamente (Valores de Aguas Andinas del 5 de Septiembre de 2015), y el precio del agua potable sin flúor es de $0,53 \mathrm{US} \$ / \mathrm{m}^{3}$ (a 15 de Octubre de 2015). Se estableció además un precio de venta del agua de $0,35 \mathrm{US} \$ / \mathrm{m}^{3}$, para asegurar su venta, al ser un precio más bajo que el del agua potable comprada por las municipalidades. El resultado de los cálculos realizados se muestra en la Tabla 4. Con esta alternativa serían necesarios 5 años para recuperar la inversión realizada, con un TIR del 34\% y un VAN de US\$ 44.951,21.

Además del cálculo de los principales parámetros económicos, se realizó también un análisis de sensibilidad con el objetivo de estudiar la influencia del precio del agua, el precio de la venta del agua, la energía y los impuestos sobre el valor del VAN. Para esto se establecieron unos precios mínimos,

Tabla 4. Cantidades anuales de ahorro de agua, tratamiento y venta de agua.

\begin{tabular}{|l|c|}
\hline \multicolumn{1}{|c|}{ Variable } & Coste anual (US\$) \\
\hline Ahorro de agua & 614,29 \\
\hline Ahorro de tratamiento & $13.495,11$ \\
\hline Ingresos por venta de agua & $3.130,37$ \\
\hline
\end{tabular}


Tabla 5. Precios mínimos, máximos y probables de las variables analizadas.

\begin{tabular}{|l|c|c|c|}
\hline \multicolumn{1}{|c|}{ Variable } & Precio mínimo & Precio probable & Precio máximo \\
\hline Precio del agua potable $\left(\mathrm{US} \$ / \mathrm{m}^{3}\right)$ & 0,43 & 0,58 & 0,72 \\
\hline Precio venta de agua tratada para riego $\left(\mathrm{US} \$ / \mathrm{m}^{3}\right)$ & 0,29 & 0,36 & 0,50 \\
\hline Precio de la energía $(\mathrm{US} \$ / \mathrm{kW} \times \mathrm{h})$ & 0,07 & 0,10 & 0,14 \\
\hline Impuesto $(\%)$ & 17 & 21 & 27 \\
\hline
\end{tabular}

máximos y más probables de cada una de ellas, recogidos en la Tabla 5.

El resultado del análisis de sensibilidad de todas las variables sobre el valor del VAN indica que este será positivo con una probabilidad del $100 \%$, y que el rango de variación estará entre los US\$ 40.345,82 y los US\$ 51.873,20 (Figura 7(A)). La variable con más influencia sobre el valor del VAN es el precio fijado para la venta del agua, con un efecto positivo en la sensibilidad del $90,5 \%$ mientras que el precio del agua, la energía y los impuestos tienen un efecto menor (Figura 7(B)).

\section{Modelo de negocio 2: Infiltración del agua en las napas subterráneas.}

En el caso de que las municipalidades no estén interesadas en la compra del agua tratada, se optaría por su infiltración en las napas subterráneas. Para este fin sería necesario asegurar que la concentración de nitrógeno de salida no sea mayor que $10 \mathrm{mg} / \mathrm{L}$, valor límite de emisión [28]. Por este motivo se consideró que el sistema granular aerobio operaría a aun TRC de $15 \mathrm{~d}$ con el fin de garantizar el desarrollo de bacterias nitrificantes. Esto implicaría un gasto adicional de $3 \mathrm{~kg}$ de oxígeno diarios, lo que supone un aumento de los costes de operación anuales de US\$ 94,00.

Con esta alternativa el ahorro correspondiente a la compra de agua para regadío y al tratamiento del agua residual siguen siendo los mismos. En este caso se supone que el gobierno pagaría por la infiltración del agua, al tratarse de un beneficio ambiental, con un precio de $0,07 \mathrm{US} \$ / \mathrm{m}^{3}$. De esta forma se obtienen menos ingresos que en el caso anterior (Tabla 6). Con esta alternativa serían necesarios 6 años para recuperar la inversión, más tiempo que en el caso anterior, el TIR sería del $25 \%$ y el VAN US\$ 27.432,22.

Para el análisis de sensibilidad se consideraron las mismas variables que en el anterior modelo,
(A)

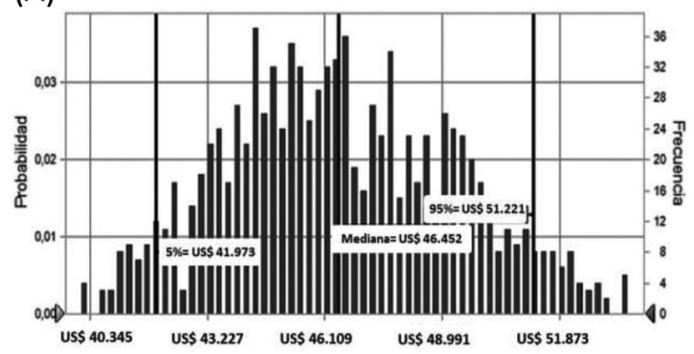

(B)

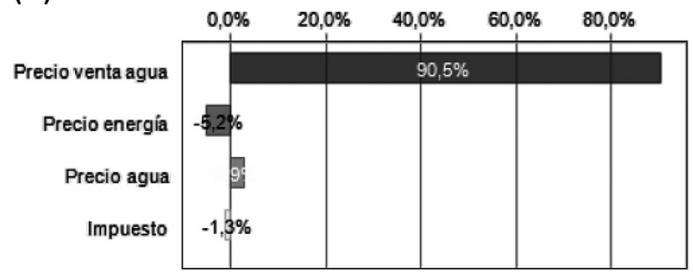

Figura 7. Modelo de negocio 1: (A) Análisis de sensibilidad de todas las variables sobre el VAN. (B) Influencia de las distintas variables sobre el VAN.

Tabla 6. Cantidades anuales de ahorro de agua, tratamiento y venta de agua.

\begin{tabular}{|l|c|}
\hline \multicolumn{1}{|c|}{ Variable } & $\begin{array}{c}\text { Coste anual } \\
\text { (US\$) }\end{array}$ \\
\hline Ahorro de agua & 614,29 \\
\hline Ahorro de tratamiento & $13.495,11$ \\
\hline Ingresos por infiltración de agua & 652,16 \\
\hline
\end{tabular}

cambiando el precio de venta del agua tratada por el precio que el gobierno pagaría por la infiltración de la misma, como se muestra en la Tabla 7.

El resultado del análisis de sensibilidad de todas las variables (Figura 8(A)), indica que el VAN siempre va a tener un valor positivo, con un rango de variación entre US\$25.072,05 y US\$27.393,38. El parámetro 
Tabla 7. Precios mínimo, máximo y probable de las variables analizadas.

\begin{tabular}{|l|c|c|c|}
\hline \multicolumn{1}{|c|}{ Variable } & Precio mínimo & Precio probable & Precio máximo \\
\hline Precio del agua $\left(\mathrm{US} \$ \mathrm{~m}^{3}\right)$ & 0,43 & 0,58 & 0,72 \\
\hline Precio pago del gobierno $\left(\mathrm{US} \$ / \mathrm{m}^{3}\right)$ & 0,07 & 0,08 & 0,09 \\
\hline Precio de la energía $(\mathrm{US} \$ \mathrm{~kW} \times \mathrm{h})$ & 0,07 & 0,10 & 0,14 \\
\hline Impuesto $(\%)$ & 17 & 21 & 27 \\
\hline
\end{tabular}

con mayor influencia en la variación del VAN es el precio de la energía, que afecta negativamente, mientras que el resto de parámetros tienen un efecto positivo y de menor impacto (Figura 8(B)).

\section{CONCLUSIONES}

Los sistemas de biomasa granular son una tecnología factible para tratar aguas residuales urbanas procedentes de condominios que se puede implementar en espacios reducidos.

El análisis de los modelos de negocio propuestos muestra que el uso del agua tratada para riego en el propio condominio combinado con la venta del excedente a las municipalidades o su infiltración en las napas subterráneas, subvencionada por el Gobierno, son alternativas económicamente viables. Sin embargo la primera opción representaría un

(A)

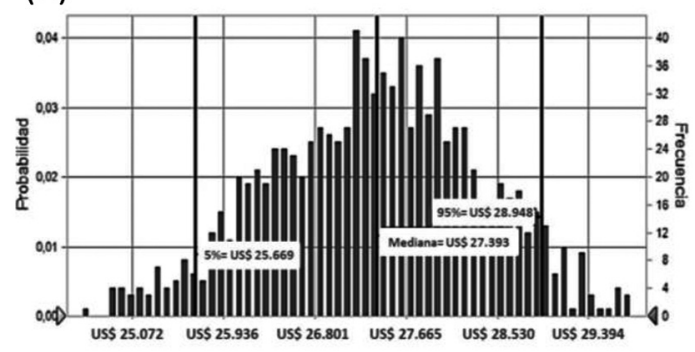

(B)

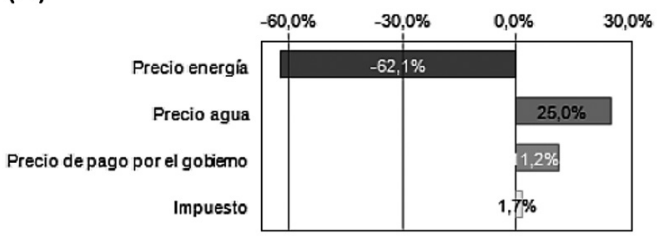

Figura 8. Modelo de negocio 2: (A) Análisis de sensibilidad de todas las variables sobre el VAN. (B) Influencia de las distintas variables sobre el VAN. escenario más favorable al ser necesarios 5 años para recuperar la inversión, con un TIR del 34\% y un VAN de US\$ 44.951,21, mientras que en la segunda opción serían necesarios 6 años para recuperar la inversión, el TIR sería del $25 \%$ y el VAN US\$ 27.432,22.

Además el análisis de sensibilidad del primer modelo de negocio muestra que el precio de venta del agua tratada es el factor que más influencia la variación del VAN, teniendo siempre un efecto positivo del $90,5 \%$. En el segundo modelo de negocio el precio de la energía es el que más afecta al VAN, pero en este caso se trata de una influencia negativa, contrayendo su valor un $62,1 \%$.

\section{AGRADECIMIENTOS}

Esta investigación fue financiada por los proyectos del gobierno Chileno: FONDECYT regular 1180650 y ANID/FONDAP/15130015; y del gobierno Español: FISHPOL (CTQ2014-55021-R) y GRANDSEA (CTM2014-55397-JIN). Los autores de la UPLA agradecen a: Programa de Fondo Desarrollo Disciplinario (18.105) de la UPLA, y LABMAI de la UPLA. M. Belmonte pertenece al Grupo de Investigación UPLAguas. Los autores de la USC pertenecen a CRETUS (AGRUP2015-02) y al Grupo Gallego de Investigación Competitiva (GRC ED431C 2017/29). J.L. Campos pertenece al Centro UAI Earth.

\section{REFERENCIAS}

[1] G. Libralato, A.V. Ghirardini and F. Avezzù F. "To centralise or to decentralise: An overview of the most recent trends in wastewater treatment management". Journal of Environmental Management. Vol. $94 \mathrm{~N}^{\mathrm{o}} 1$, pp. 61-68. February, 2012. ISSN: 0301-4797. DOI: 10.1016/j.jenvman.2011.07.010

[2] D. Gerrity, B. Pecson, R.S. Trussell and R.R. Trussell. "Potable reuse treatment trains 
throughout the world". Journal of Water Supply: Research and Technology. Vol. 62 $\mathrm{N}^{\circ}$ 6, pp. 321-338. September, 2013. ISSN: 1606-9935. DOI: 10.2166/aqua.2013.041

[3] M.A. Massoud, A. Tarhini and J.A. Nasr. "Decentralized approaches to wastewater treatment and management: Applicability in developing countries". Journal of Environmental Management. Vol. $90 \mathrm{~N}^{\mathrm{o}} 1$, pp. 652-659. January, 2009. ISSN: 03014797. DOI: $10.1016 /$ j.jenvman.2008.07.001

[4] A. Vega, K. Lizama and P. Pastén "Water Quality: Trends and Challenges". In: Water Policy in Chile. Springer, Berlin, Germany. pp. 219. 2018. ISBN 978-3-319-76702-4.

[5] M.Y. Sklarz, A. Gross, A. Yakirevich and M. Soares. "A recirculating vertical flow constructed wetland for the treatment of domestic wastewater". Desalination Vol. 246 $\mathrm{N}^{\mathrm{o}} 1$-3, pp. 617-624. September 2009. ISSN: 0011-9164. DOI: 10.1016/j.desal.2008.09.002

[6] R. Mancilla, J. Zúñiga, E. Salgado, M. Schiappacasse and R. Chamy. "Constructed wetlands for domestic wastewater treatment in a Mediterranean climate region in Chile". Electronic Journal of Biotechnology, Vol. 16 $\mathrm{N}^{\circ}$ 4, pp. 1-13. July, 2013. ISSN 0717-3458. DOI: $10.2225 /$ vol16-issue4-fulltext-5

[7] C.A. Villamar, I. Vera-Puerto, D. Rivera and F. De la Hoz. "Reuse and Recycling of Livestock and Municipal Wastewater in Chilean Agriculture: A Preliminary Assessment". Water, Vol. $10 \mathrm{~N}^{\circ}$ 6, pp. 817. June, 2018 ISSN: 2073-4441. DOI: 10.3390/ w10060817

[8] I. Vera, C. Jorquera, D. López y G. Vidal. "Humedales construidos para tratamiento y reúso de aguas servidas en Chile: reflexiones". Tecnología y Ciencias del Agua, Vol. $7 \mathrm{~N}^{\circ} 3$, pp. 19-35. Mayo-junio, 2016. ISSN: 2007-2422.

[9] A. Val del Río, M. Figueroa, B. Arrojo, A. Mosquera-Corral, J.L. Campos, G. GarcíaTorriello and R. Méndez. "Aerobic granular SBR systems applied to the treatment of industrial effluents". Journal of Environmental Management. Vol. 95 Suplementary, pp. 88-92. March, 2012. ISSN: 0301-4797. DOI: 10.1016/j.jenvman.2011.03.019.

[10] Z.H. Li, T. Kuba and T. Kusuda. "Aerobic granular sludge: a promising technology for decentralised wastewater treatment".
Water Science and Technology. Vol. $53 \mathrm{~N}^{\circ} 9$, pp. 79-85. May, 2006. ISSN: 0273-1223. DOI: $10.2166 /$ wst.2006.278.

[11] A. Mosquera-Corral, A. Val del Río, H. Moralejo-Gárate, A. Sánchez, R. Méndez and J.L. Campos. "The aerobic granulation as an alternative to the conventional activated sludge process". IWA Publishing. First Edition. Londres, Reino Unido, pp. 96-113. 2015. ISBN: 9781780405018.

[12] L.M.M. de Bruin, M.K. de Kreuk, H.F.R. van der Roest, C. Uijterlinde and M.C.M. van Loosdrecht. "Aerobic granular sludge technology: an alternative to activated sludge?". Water Science and Technology. Vol. $49 \mathrm{~N}^{\circ} 11-12$, pp. 1-7. June, 2004. ISSN: 0273-1223. URL: http://wst.iwaponline.com/ content/49/11-12/1

[13] S. Bengtsson, M. de Blois, B.M. Wilén and D. Gustavsson "A comparison of aerobic granular sludge with conventional and compact biological treatment technologies". Environmental Technology. ISSN: 0959-3330. DOI: 10.1080/09593330.2018.1452985

[14] B. Ni, W. Xie, S. Liu, H. Yu, Y. Wang, G. Wang and X. Dai. "Granulation of activated sludge in a pilot-scale sequencing batch reactor for the treatment of low-strength municipal wastewater". Water Research. Vol. 43 No 3 , pp. 751-761. February, 2009. ISSN: 00431354. DOI: 10.1016/j.watres.2008.11.009

[15] M. Pronk, B. de Bruin, P. Kamminga, R. Kleerebezem and M.C.M. van Loosdrecht. "Full scale performance of the aerobic granular sludge process for sewage treatment". Water Research. Vol. 84 N $^{\circ} 1$, pp. 207-217. November, 2009. ISSN: 0043-1354. DOI: 10.1016/j.watres.2015.07.011

[16] J. Anderson. "The environmental benefits of water recycling and reuse". Water Science and Technology: Water Supply. Vol. $3 \mathrm{~N}^{\circ} 4$, pp. 1-10. August, 2003. ISSN: 1606-9749. URL: http://ws.iwaponline.com/content/3/4/1

[17] K.J. Jungles, M. Figueroa, N. Morales, A. Val del Río, R.H. Ribeiro da Costa, J.L. Campos, A. Mosquera-Corral and R. Méndez. "Start up of a pilot scale aerobic granular reactor for organic matter and nitrogen removal". Journal of Chemical. Technology and Biotechnology. Vol. $86 \mathrm{~N}^{\circ}$ 5, pp. 763-768. May, 2011. ISSN: 1097-4660. DOI: $10.1002 /$ jctb.2589. 
[18] APHA-AWWA-WPCF. "Standard methods for the examination of water and wastewater". American Public Health Association/ American Water Works Association/ Water Environment Federation. 21st ed. Washington DC, EUA, pp. 1496. 2005. ISBN: 978-0875530475.

[19] N. Corral. "Modelo para el diseño y evaluación del metabolismo circular de comunidades incorporando tecnologías limpias". Memoria de Tesis. Universidad Adolfo Ibáñez. Santiago, Chile, pp. 164. 2014.

[20] N. Morales, A. Val del Río, J. R. VázquezPadín, R. Méndez, A. Mosquera-Corral and J.L. Campos. "Integration of the Anammox process to the rejection water and mainstream lines of WWTPs". Chemosphere. Vol. 140, pp. 99-105. December, 2015. ISSN: 0045-6535. DOI: 10.1016/j.chemosphere. 2015.03.058

[21] Remosa. "Regeneración/Reutilización de aguas, Depuración de aguas residuales domésticas, Separadores de hidrocarburos, Almacenamiento de líquidos [en línea]". 2014. Última consulta: 25/03/2018. URL: http://www.remosa.net

[22] USEPA. EPA 832-F-00-016. "Wastewater Technology Fact Sheet Package Plants. Office of Water, United State Environmental Protection Agency. Manual”, pp. 12. Washington, D.C., EUA. 2000.
[23] M.S. Peters and K.D. Timmerhaus. "Plant Design and Economics for Chemical Engineers". McGraw Hill. 4th ed. Nueva York, EUA, pp. 910. 1991. ISBN: 978-0070496132.

[24] J. Couper, W. Penny and J. Fair. "Chemical Process Equipment". Selection and Design. Gulf Professional Publishing, 2a ed. Oxford, United Kingdom, pp. 832. 2009. ISBN: 978-0123725066.

[25] D.G. Weissbrodt, S. Lochmatter, S. Ebrahimi, P. Rossi, J. Maillard and C. Holliger. "Bacterial selection during the formation of early-stage aerobic granules in wastewater treatment systems operated under wash-out dynamics". Frontiers in Microbiology. Vol. 3, pp. 1-22. September, 2012. ISSN: 1664-302X. DOI: 10.3389/fmicb.2012.00332.

[26] J. Barton, C. Pavez, S. Reyes and F. Salinas. "Consumo de agua para riego de áreas verdes: Parques urbanos". Centro UC cambio global. Santiago de Chile, Chile, pp. 55. 2013.

[27] R. Astaburuaga. "El agua en las zonas áridas de Chile". ARQ. Vol. 57, pp. 68-73. Julio, 2004. ISSN: 0717-6996. DOI: http://dx.doi. org/10.4067/S0717-69962004005700018

[28] CONAMA. "Decreto Supremo 46/03, Norma de emisión de residuos líquidos a aguas subterráneas". Comisión Nacional del Medio Ambiente, Gobierno de Chile. Biblioteca del Congreso Nacional de Chile. 17 de enero de 2003. 